\title{
Spherical Harmonic Analysis and Model-Limited Extrapolation on the Sphere: Integral Equation Formulation
}

\author{
Rodney A. Kennedy, Wen Zhang and Thushara D. Abhayapala
}

\begin{abstract}
The classical problem of extrapolation of a bandlimited signal from limited time domain data is revisited for signals defined on the sphere. That is, given limited or incomplete measurements of an isotropic low pass signal on the unit sphere, $\mathbb{S}^{2}$, find the unique extrapolation to the complete unit sphere. Signals defined on the unit sphere arise in a number of applications, such as beampatterns in azimuth and elevation and head related transfer functions. Our investigations explore the role of integral equation operators in characterizing the extrapolation problem which leads to an iterative algorithm analogous to that obtained in the time-frequency case.
\end{abstract}

\section{INTRODUCTION}

A fundamental problem in signal processing is that of extrapolation of bandlimited signals from incomplete time domain data. That is, knowing a signal on a finite time interval on the real line, the objective is to determine the signal on the doubly infinite real line. At a conceptual mathematical level this is a trivial task because it is merely as instance of analytic continuation. However, the engineering challenge is to find a robust and simple algorithm for this problem. Papoulis gave an algorithm for this task and characterized its convergence [1]. It was show that fundamental work of Slepian et al. [2], [3] emerged naturally in characterizing the problem.

A bandlimited signal is subject to a smoothness constraint (usually low pass but equally well bandpass and other cases which can be handled). In carrying over the notion of "bandlimited" to the sphere the objective is to find a suitable lowpass characterization. This is well-known and involves truncating the spherical harmonic expansion of the signal on the sphere retaining the lowest order terms [4], [5]. The spherical harmonics do involve two indices and we impose the condition that the low pass action is isotropic on the sphere and this implies that the truncation of the spherical harmonics takes a particular but very natural form where only one of the indices need be limited. It can be shown that for isotropic filtering (isotropic spherical convolution) it is sufficient to consider purely real valued functions on the sphere. In our development below we retain the use of complex representations so as to, in-principle, deal with generalization to directional (nonisotropic) smoothing on the sphere.

At an abstract level there is little difference between the time/frequency case ("time-case") and the sphere-

Rodney A. Kennedy, Wen Zhang and Thushara D. Abhayapala are with the Department of Information Engineering, Research School of Information Sciences and Engineering, The Australian National University rodney.kennedyeanu.edu.au spatial/spherical harmonic case ("sphere-case") which guarantees a set of meaningful results for the sphere-case. However, the sphere-case is in some aspects more challenging than the time-case but in others less so. It is helpful to flag these aspects. Firstly, the sphere-case is multi-variate and the analog of the "intervals" of the time-case are "regions" in the sphere-case which can be varied in shape and not necessarily simply connected. The theory for the sphere-case is valid for all sufficiently regular regions, and the shape of the region has a significant role to play in characterizing the problem (the classical boundary value problem in partial differential equations shows a similar very significant role of the boundary in the formulation). In contrast, the interval in the time-case is relatively benign. Secondly, in the sphere-case, the unit sphere is a twice periodic and finite structure, and this means the Fourier representation is discrete, that is, harmonic (as the name spherical harmonics suggests). This is simpler than the time-case where the Fourier transform is continuous. Were the time-case signal finite in duration or periodic then this is a better analog of the sphere-case. Finally, in the spherecase, when truncating the spherical harmonic expansion (low pass) the resulting signal is finite dimensional which means that standard linear algebra can be used to characterize the extrapolating functions [5], [6]. However, other signal models which would leave an infinite number of spherical harmonic coefficients, such as a high pass isotropic filter, are not finite dimensional. For this result in this paper we provide an alternative formulation that covers such generalizations and does not rely on finite dimensional linear algebra.

In motivating the consideration of signals on a sphere we mention a few applications: i) reconstructing antenna beampatterns from incomplete data on the sphere [7]; ii) reconstructing reconstructing head related transfer functions (transfer functions which are a function of the azimuth and elevation as well as frequency) [6]; and iii) geophysical, planetary and cosmological applications [5].

Closely related to this work, but not formally treated here, are results for determining optimally concentrated functions for the sphere and ball (sphere plus interior). Just like the time/frequency case there are notions of signal concentration that can be developed for the sphere spatial caseconcentration of functions on the sphere [5] and concentration of functions in ball shaped domain subject to a wave equation condition [4]. 


\section{PROBLEM FORMULATION}

The problem being addressed is: Given limited or incomplete measurements of an isotropic low pass signal on the unit sphere, $\mathbb{S}^{2}$, find the unique extrapolation to the complete unit sphere. More precisely if the signal is available only on some subregion $\Gamma \subset \mathbb{S}^{2}$ and is known to be isotropically low pass (spatially bandlimited), determine the signal on the $\mathbb{S}^{2} \backslash \Gamma$.

\section{A. Fredholm Integral Equation}

A general inhomogeneous Fredholm integral equation of the first kind takes the form

$$
(\mathscr{L} f)(\boldsymbol{x})=\int_{\Omega} L(\boldsymbol{x}, \boldsymbol{y}) f(\boldsymbol{y}) d \boldsymbol{y}, \quad \boldsymbol{x} \in \Omega
$$

where $\Omega$ is the domain of interest and $L(\boldsymbol{x}, \boldsymbol{y})$ is the kernel. The kernel will be taken as Hilbert-Schmidt unless otherwise stated, meaning

$$
\|\mathscr{L}\|^{2}=\iint_{\Omega \times \Omega}|L(\boldsymbol{x}, \boldsymbol{y})|^{2} d \boldsymbol{y} d \boldsymbol{x}<\infty, \quad \boldsymbol{x}, \boldsymbol{y} \in \Omega .
$$

The integral equation (1) has adjoint

$$
\left(\mathscr{L}^{*} f\right)(\boldsymbol{x})=\int_{\Omega} \overline{L(\boldsymbol{y}, \boldsymbol{x})} f(\boldsymbol{y}) d \boldsymbol{y}, \quad \boldsymbol{x} \in \Omega .
$$

The operator in (1) is self-adjoint, $\mathscr{L}^{*}=\mathscr{L}$, whenever $L(\boldsymbol{x}, \boldsymbol{y})=\overline{L(\boldsymbol{y}, \boldsymbol{x})}$. Condition (2) is sufficient to make the integral operator compact.

In the following, we shall be considering functions on the unit sphere, $\mathbb{S}^{2}$, or a subregion (closed bounded region) of the unit sphere, $\Gamma \subset \mathbb{S}^{2}$.

\section{B. Spherical Harmonics}

Notation: In the following, we use the shorthand

$$
\sum_{m, n} \triangleq \sum_{n=0}^{\infty} \sum_{m=-n}^{n} \text { and } \sum_{m,|n|<N} \triangleq \sum_{n=0}^{N} \sum_{m=-n}^{n}
$$

The spherical harmonics, as a function of $\boldsymbol{x}=(\theta, \phi)$ (representing a direction in space as a vector on the unit sphere),

$$
\begin{aligned}
& Y_{n}^{m}(\boldsymbol{x}) \triangleq \sqrt{\frac{2 n+1}{4 \pi} \frac{(n-|m|) !}{(n+|m|) !}} P_{n}^{|m|}(\cos \theta) e^{i m \phi}, \\
& n=0,1,2, \ldots, m=-n, \ldots, n
\end{aligned}
$$

where $P_{n}^{m}$ are the associated Legendre functions, defined for $m=0,1, \ldots, n$, which form a complete orthonormal system with respect to the natural inner product on the 2-sphere $\mathbb{S}^{2}$ [8]. We denote this complex Hilbert Space as $L^{2}\left(\mathbb{S}^{2}\right)$. For any $f \in$ $L^{2}\left(\mathbb{S}^{2}\right)$ we have the Spherical Harmonic Transform identity

$$
f(\boldsymbol{x})=\sum_{m, n}\left(\int_{\mathbb{S}^{2}} f(\boldsymbol{y}) \overline{Y_{n}^{m}(\boldsymbol{y})} d \boldsymbol{y}\right) Y_{n}^{m}(\boldsymbol{x})
$$

where the equality is understood in terms of convergence in the mean [9]. The complex coefficients in (6) are the generalized complex Fourier coefficients and are given by

$$
\begin{aligned}
F_{n}^{m} \triangleq \int_{\mathbb{S}^{2}} f(\boldsymbol{y}) \overline{Y_{n}^{m}(\boldsymbol{y})} d \boldsymbol{y}, \\
\quad n=0,1,2, \ldots, m=-n, \ldots, n
\end{aligned}
$$

\section{Two Projections on the Sphere}

Mode Limiting Operator: Given the Spherical Harmonic Expansion, (6), we define a mode limiting operator, $\mathscr{B}_{N}$, on Hilbert Space $L^{2}\left(\mathbb{S}^{2}\right)$, as the truncated Spherical Harmonic Transform (6),

$$
\left(\mathscr{B}_{N} f\right)(\boldsymbol{x}) \triangleq \sum_{m,|n|<N}\left(\int_{\mathbb{S}^{2}} f(\boldsymbol{y}) \overline{Y_{n}^{m}(\boldsymbol{y})} d \boldsymbol{y}\right) Y_{n}^{m}(\boldsymbol{x})
$$

which is an isotropic low pass filter on $\mathbb{S}^{2}$. A simple manipulation reveals (8) to be an integral operator of the form (1) with hermitian symmetric kernel

$$
B_{N}(\boldsymbol{x}, \boldsymbol{y}) \triangleq \sum_{m,|n|<N} Y_{n}^{m}(\boldsymbol{x}) \overline{Y_{n}^{m}(\boldsymbol{y})} .
$$

Using a well-known identity, this kernel can be simplified to

$$
B_{N}(\boldsymbol{x}, \boldsymbol{y}) \triangleq \sum_{n=0}^{N-1} \frac{2 n+1}{4 \pi} P_{n}(\boldsymbol{x} \cdot \boldsymbol{y})
$$

where $\boldsymbol{x} \cdot \boldsymbol{y}$ is the inner product and equals $\cos \zeta$ where $\zeta$ denotes the angle between $\boldsymbol{x}$ and $\boldsymbol{y}$, [8]. This reveals that the kernel is actually purely real. Operator $\mathscr{B}_{N}$ is a finite rank operator of dimension $N^{2}$ and hence compact [10]. It is also a projection, idempotent and self adjoint.

Note that a kernel on the unit sphere that can be expressed as a function of $\boldsymbol{x} \cdot \boldsymbol{y}$ is a type of isotropic spherical convolutionthe analog on the unit sphere for a standard convolution kernel on the real line which is a function of $\boldsymbol{x}-\boldsymbol{y}$.

Truncation Operator: The second operator is the truncation operator, $\mathscr{D}_{\Gamma}$, defined as

$$
\left(\mathscr{D}_{\Gamma} f\right)(\boldsymbol{x})= \begin{cases}f(\boldsymbol{x}) & \boldsymbol{x} \in \Gamma \\ 0 & \boldsymbol{x} \in \Omega \backslash \Gamma\end{cases}
$$

for some 2D subregion (closed bounded region) $\Gamma \subset \Omega$. Again this can be expressed as an integral operator of the form (1) using generalized functions, leading to a kernel of the form

$$
D_{\Gamma}(\boldsymbol{x}, \boldsymbol{y}) \triangleq \chi_{\Gamma}(\boldsymbol{y}) \delta(\boldsymbol{x}-\boldsymbol{y})
$$

where $\chi_{\Gamma}(\cdot)$ denotes the characteristic (indicator) function of the region $\Gamma$, and $\delta(\cdot)$ is the 2D Dirac delta function. This kernel is real and satisfies $D_{N}(\boldsymbol{x}, \boldsymbol{y})=D_{N}(\boldsymbol{y}, \boldsymbol{x})$ and so the operator is self adjoint. It is idempotent and a projection but it is not compact. Nor is it Hilbert-Schmidt (as that would imply it were compact), that is, it does not satisfy the sufficient condition (2). Equivalently, we can write the truncation operator as a multiplication operator

$$
\left(\mathscr{D}_{\Gamma} f\right)(\boldsymbol{x})=\chi_{\Gamma}(\boldsymbol{x}) f(\boldsymbol{x})
$$

using the characteristic function. 
Operator Compositions: The two compact projection operators, on $L^{2}\left(\mathbb{S}^{2}\right), \mathscr{B}_{N}$ and $\mathscr{D}_{\Gamma}$ do not commute. This lack of commutativity implies neither composite operator $\mathscr{B}_{N} \circ \mathscr{D}_{\Gamma}$ nor composite operator $\mathscr{D}_{\Gamma} \circ \mathscr{B}_{N}$ are self adjoint on $L^{2}\left(\mathbb{S}^{2}\right)$ [10]. Indeed the integral equation kernel for the composite operator

$$
\mathscr{C}_{N, \Gamma} \triangleq \mathscr{B}_{N} \circ \mathscr{D}_{\Gamma}
$$

on $L^{2}\left(\mathbb{S}^{2}\right)$ takes the form

$$
\begin{aligned}
C_{N, \Gamma}(\boldsymbol{x}, \boldsymbol{y}) & \triangleq \chi_{\Gamma}(\boldsymbol{y}) \sum_{m,|n|<N} Y_{n}^{m}(\boldsymbol{x}) \overline{Y_{n}^{m}(\boldsymbol{y})} \\
& \neq \overline{C_{N, \Gamma}(\boldsymbol{y}, \boldsymbol{x})},
\end{aligned}
$$

that is, it is not symmetric for all $\boldsymbol{x}, \boldsymbol{y} \in \mathbb{S}^{2}$, [11]. Similarly, the composite operator

$$
\mathscr{C}_{\Gamma, N} \triangleq \mathscr{D}_{\Gamma} \circ \mathscr{B}_{N}
$$

on $L^{2}\left(\mathbb{S}^{2}\right)$ has kernel

$$
\begin{aligned}
C_{\Gamma, N}(\boldsymbol{x}, \boldsymbol{y}) & \triangleq \chi_{\Gamma}(\boldsymbol{x}) \sum_{m,|n|<N} Y_{n}^{m}(\boldsymbol{x}) \overline{Y_{n}^{m}(\boldsymbol{y})} \\
& \neq \overline{C_{\Gamma, N}(\boldsymbol{y}, \boldsymbol{x})} .
\end{aligned}
$$

We also observe that $\mathscr{D}_{\Gamma} \mathscr{B}_{N}$ is the adjoint of $\mathscr{B}_{N} \mathscr{D}_{\Gamma}$, and this is equivalent to

$$
C_{\Gamma, N}(\boldsymbol{x}, \boldsymbol{y})=\overline{C_{N, \Gamma}(\boldsymbol{y}, \boldsymbol{x})} .
$$

Lack of self-adjointness means we cannot appeal directly to the Spectral Theorem for compact, self adjoint operators, to characterize them.

Spectral Characterization: By restricting the domain of these operators and their compositions we can appeal to the Spectral Theorem for compact, self adjoint operators. As an operator on $L^{2}(\Gamma)$, the composite operator $\mathscr{C}_{N, \Gamma} \triangleq \mathscr{B}_{N} \mathscr{D}_{\Gamma}$ can be shown to be self adjoint, as follows.

Consider (6) which holds for any $f \in L^{2}\left(\mathbb{S}^{2}\right)$ and so holds for the truncated function $\left(\mathscr{D}_{\Gamma} f\right)(\boldsymbol{x}) \in L^{2}\left(\mathbb{S}^{2}\right)$, that is,

$$
\begin{aligned}
\left(\mathscr{D}_{\Gamma} f\right)(\boldsymbol{x}) & =\sum_{m, n}\left(\int_{\mathbb{S}^{2}}\left(\mathscr{D}_{\Gamma} f\right)(\boldsymbol{y}) \overline{Y_{n}^{m}(\boldsymbol{y})} d \boldsymbol{y}\right) Y_{n}^{m}(\boldsymbol{x}) \\
& =\sum_{m, n}\left(\int_{\mathbb{S}^{2}} \chi_{\Gamma}(\boldsymbol{y}) f(\boldsymbol{y}) \overline{Y_{n}^{m}(\boldsymbol{y})} d \boldsymbol{y}\right) Y_{n}^{m}(\boldsymbol{x}) \\
& =\sum_{m, n}\left(\int_{\Gamma} f(y) \overline{Y_{n}^{m}(\boldsymbol{y})} d \boldsymbol{y}\right) Y_{n}^{m}(\boldsymbol{x})
\end{aligned}
$$

valid for all $\boldsymbol{x} \in \mathbb{S}^{2}$. However, when $\boldsymbol{x}$ is restricted to $\Gamma \subset \mathbb{S}^{2}$, $\mathscr{D}_{\Gamma} f=f$, and we have

$$
\begin{aligned}
f(\boldsymbol{x}) & =\sum_{m, n}\left(\int_{\Gamma} f(\boldsymbol{y}) \overline{Y_{n}^{m}(\boldsymbol{y})} d \boldsymbol{y}\right) Y_{n}^{m}(\boldsymbol{x}), \boldsymbol{x} \in \Gamma \\
& =\int_{\Gamma} B(\boldsymbol{x}, \boldsymbol{y}) f(\boldsymbol{y}) d \boldsymbol{y}, \boldsymbol{x} \in \Gamma
\end{aligned}
$$

where, as a functional on $\Gamma \times \Gamma$,

$$
\begin{aligned}
B(\boldsymbol{x}, \boldsymbol{y}) & \triangleq \sum_{m, n} Y_{n}^{m}(\boldsymbol{x}) \overline{Y_{n}^{m}(\boldsymbol{y})} \\
& =\sum_{n=0}^{\infty} \frac{2 n+1}{4 \pi} P_{n}(\boldsymbol{x} \cdot \boldsymbol{y}) \\
& =\delta(\boldsymbol{x}-\boldsymbol{y})
\end{aligned}
$$

which simply is the completeness relation for spherical harmonics-operator $\mathscr{D}_{\Gamma}$ is equivalent to the identity operator on $L^{2}(\Gamma)$.

Fundamental Integral Equation: Next we apply modelimiting operator $\mathscr{B}_{N}$ to $\mathscr{D}_{\Gamma} f=f$ in (24) to obtain our first fundamental integral equation

$$
\left(\mathscr{B}_{N} f\right)(\boldsymbol{x})=\int_{\Gamma} B_{N}(\boldsymbol{x}, \boldsymbol{y}) f(\boldsymbol{y}) d \boldsymbol{y}, \boldsymbol{x} \in \Gamma
$$

where

$$
B_{N}(\boldsymbol{x}, \boldsymbol{y})=\sum_{n=0}^{N-1} \frac{2 n+1}{4 \pi} P_{n}(\boldsymbol{x} \cdot \boldsymbol{y}), \quad \boldsymbol{x}, \boldsymbol{y} \in \Gamma
$$

is the kernel previously obtained in (9) but now defined on domain $\Gamma \times \Gamma$. Due to the symmetry of the kernel, the integral operator (29) on $L^{2}(\Gamma)$ is self adjoint (as well as compact).

Given the integral equation corresponding to mode-limiting on $L^{2}(\Gamma)$ is compact and self-adjoint, then by the Spectral Theorem, it has a fully defined discrete spectral representation in the form

$$
\begin{array}{r}
\left(\mathscr{B}_{N} \phi_{k}\right)(\boldsymbol{x})=\lambda_{k} \phi_{k}(\boldsymbol{x}), \boldsymbol{x} \in \Gamma, \\
k=1,2,3, \ldots
\end{array}
$$

where $\left\{\phi_{k}(\boldsymbol{x}): \boldsymbol{x} \in \Gamma\right\}_{k=1}^{\infty}$ are eigenfunctions ${ }^{1}$ with $\left\{\lambda_{k}\right\}_{k=1}^{\infty}$ the corresponding real eigenvalues (these eigenvalues are positive and bounded above by unity since the operator is a projection). This eigenfunction equation, (31), can be rewritten

$$
\begin{aligned}
\phi_{k}(\boldsymbol{x})=\frac{1}{\lambda_{k}} \int_{\Gamma} B_{N}(\boldsymbol{x}, \boldsymbol{y}) \phi_{k}(\boldsymbol{y}) d \boldsymbol{y}, & \boldsymbol{x} \in \Gamma \\
k & =1,2,3, \ldots
\end{aligned}
$$

By the Spectral Theorem, these eigenfunctions can be chosen to form a complete orthogonal set on the Hilbert Space $L^{2}(\Gamma)$, [9]. Normally the orthogonal eigenfunctions $\left\{\phi_{k}(\boldsymbol{x}): \boldsymbol{x} \in \Gamma\right\}_{k=1}^{\infty}$ are scaled to make them orthonormal. However, as in the analogous case of Prolate Spheroidal Wave Functions [3], it is advantageous to choose the scaling in the definition of the $\left\{\phi_{k}(\boldsymbol{x}): \boldsymbol{x} \in \Gamma\right\}_{k=1}^{\infty}$ in a way that the orthogonality relation is

$$
\int_{\Gamma} \phi_{k}(\boldsymbol{x}) \overline{\phi_{\ell}(\boldsymbol{x})} d \boldsymbol{x}=\lambda_{k} \delta_{k \ell}
$$

\footnotetext{
${ }^{1}$ These are unique up to a complex scaling factor and, for each repeated eigenvalue, if any, need to be chosen to be independent.
} 
Note that suitably normalized (orthonormal) eigenfunctions can be obtained through

$$
\varphi_{k}(\boldsymbol{x}) \triangleq \frac{1}{\sqrt{\lambda_{k}}} \phi_{k}(\boldsymbol{x}), \quad \boldsymbol{x} \in \Gamma
$$

with

$$
\int_{\Gamma} \varphi_{k}(\boldsymbol{x}) \overline{\varphi_{\ell}(\boldsymbol{x})} d \boldsymbol{x}=\delta_{k \ell}
$$

Then kernel expansion, usually associated with Mercer's Theorem [9], can be expressed in two ways

$$
\begin{aligned}
& B_{N}(\boldsymbol{x}, \boldsymbol{y})=\sum_{k=1}^{\infty} \lambda_{k} \varphi_{k}(\boldsymbol{x}) \overline{\varphi_{k}(\boldsymbol{y})}, \quad \boldsymbol{x}, \boldsymbol{y} \in \Gamma \\
& B_{N}(\boldsymbol{x}, \boldsymbol{y})=\sum_{k=1}^{\infty} \phi_{k}(\boldsymbol{x}) \overline{\phi_{k}(\boldsymbol{y})}, \quad \boldsymbol{x}, \boldsymbol{y} \in \Gamma
\end{aligned}
$$

and the completeness relation takes the two forms

$$
\begin{aligned}
\sum_{k=1}^{\infty} \varphi_{k}(\boldsymbol{x}) \overline{\varphi_{\ell}(\boldsymbol{y})} & =\delta(\boldsymbol{x}-\boldsymbol{y}), \quad \boldsymbol{x}, \boldsymbol{y} \in \Gamma \\
\sum_{k=1}^{\infty} \frac{1}{\lambda_{k}} \phi_{k}(\boldsymbol{x}) \overline{\phi_{\ell}(\boldsymbol{y})} & =\delta(\boldsymbol{x}-\boldsymbol{y}), \quad \boldsymbol{x}, \boldsymbol{y} \in \Gamma
\end{aligned}
$$

The expression (32) enables us to appeal to an argument used by Slepian to extended the domain of definition of the eigenfunctions in $L^{2}(\Gamma)$, to functions in $L^{2}\left(\mathbb{S}^{2}\right)$ [2], [3]. Noting that $\phi_{k}(\cdot)$ in the integrand in (32) need only be defined on $\Gamma \subset \mathbb{S}^{2}$ for the integral to be well defined, then we can take (32) as the definition $\phi_{k}(\cdot)$ on the remainder $\mathbb{S}^{2} \backslash \Gamma$, i.e.,

$$
\begin{aligned}
\phi_{k}(\boldsymbol{x})=\frac{1}{\lambda_{k}} \int_{\Gamma} B_{N}(\boldsymbol{x}, \boldsymbol{y}) \phi_{k}(\boldsymbol{y}) d \boldsymbol{y}, & \boldsymbol{x} \in \mathbb{S}^{2} \backslash \Gamma \\
k & =1,2,3, \ldots
\end{aligned}
$$

That is, (40) is an extrapolation to the whole unit sphere $\mathbb{S}^{2}$ of the eigenfunctions of operator $\mathscr{B}_{N}$ on $L^{2}(\Gamma)$.

Next we show that the extrapolated eigenfunctions defined in (32) and (40) are in fact orthogonal on $\mathbb{S}^{2}$. Rewrite (32) and (40) using the characteristic function as

$$
\begin{array}{r}
\phi_{k}(\boldsymbol{x})=\frac{1}{\lambda_{k}} \int_{\mathbb{S}^{2}} B_{N}(\boldsymbol{x}, \boldsymbol{y})\left(\chi_{\Gamma}(\boldsymbol{y}) \phi_{k}(\boldsymbol{y})\right) d \boldsymbol{y}, \boldsymbol{x} \in \mathbb{S}^{2} \\
k=1,2,3, \ldots
\end{array}
$$

and note that the space limited function $\chi_{\Gamma}(\boldsymbol{x}) \phi_{k}(\boldsymbol{x})$ is a well-defined function in $L^{2}\left(\mathbb{S}^{2}\right)$. So (41) is a mode-limiting operation applied to $\chi_{\Gamma}(\boldsymbol{x}) \phi_{k}(\boldsymbol{x})$. This implies that the LHS of (41), $\phi_{k}(\boldsymbol{x})$, is mode-limited. However, mode-limited functions are invariant to the mode-limiting operator, that is, if $f_{N}(\boldsymbol{x}) \in L^{2}\left(\mathbb{S}^{2}\right)$ is mode-limited then

$$
\mathscr{B}_{N} f_{N}=f_{N}
$$

equivalently

$$
\int_{\mathbb{S}^{2}} B_{N}(\boldsymbol{x}, \boldsymbol{y}) f_{N}(\boldsymbol{y}) d \boldsymbol{y}=f_{N}(\boldsymbol{x})
$$

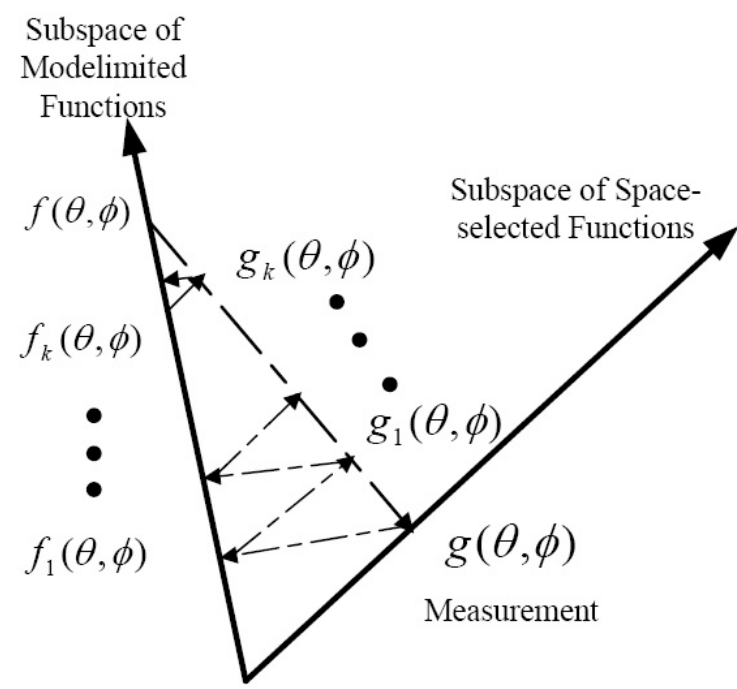

Fig. 1. Papoulis Algorithm on the Unit Sphere

Whence, for the extrapolated eigenfunctions of (31), satisfy

$$
\begin{array}{r}
\left(\mathscr{B}_{N} \phi_{k}\right)(\boldsymbol{x})=\phi_{k}(\boldsymbol{x}), \boldsymbol{x} \in \mathbb{S}^{2}, \\
k=1,2,3, \ldots
\end{array}
$$

which means they are eigenfunctions of an integral equation (44) on $L^{2}\left(\mathbb{S}^{2}\right)$ with eigenvalue 1 . This is the same operator on $L^{2}\left(\mathbb{S}^{2}\right)$ considered in (8) which is finite rank, compact and self-adjoint. It is elementary to show that the extrapolated eigenfunctions are orthonormal in the $L^{2}\left(\mathbb{S}^{2}\right)$. Clearly these extrapolated eigenfunctions are not complete in $L^{2}\left(\mathbb{S}^{2}\right)$ but are a complete orthonormal set for the subspace of mode-limited functions of order $N$.

\section{Papoulis Algorithm on the Unit Sphere}

Let $f(\boldsymbol{x})$ be the mode-limited function on the unit sphere to be determined. It satisfies

$$
f(\boldsymbol{x})=\left(\mathscr{B}_{N} f\right)(\boldsymbol{x})
$$

Let $g(\boldsymbol{x})$ be the measured function on the unit sphere which satisfies

$$
g(\boldsymbol{x})=\left(\mathscr{D}_{\Gamma} f\right)(\boldsymbol{x})
$$

where the support of $g(\boldsymbol{x})$ is $\Gamma$.

The objective of the algorithm is to reconstruct $f(\boldsymbol{x})$ for all $x \in \mathbb{S}^{2}$ from $g(\boldsymbol{x})$ which is non-zero only in $\Gamma \subset \mathbb{S}^{2}$. Towards this goal we can develop two sets of iterations, $\left\{f_{p}\right\}_{p=1}^{\infty}$ and $\left\{g_{p}\right\}_{p=1}^{\infty}$ which evolve towards $f(\boldsymbol{x})$, as shown in Fig. 1. The operator $\mathscr{D}_{\Gamma}$ defines an orthogonal projection onto the subspace of space-selected functions and the operator $\mathscr{B}_{N}$ defines an orthogonal projection onto the subspace of modelimited functions. 
Thus we have

$$
\begin{aligned}
f_{k}(\boldsymbol{x}) & =\left(\mathscr{B}_{N} g_{k-1}\right)(\boldsymbol{x}) \\
& =\left(\mathscr{B}_{N} f_{k-1}\right)(\boldsymbol{x})+\left(\mathscr{C}_{N, \Gamma}\left(f-f_{k-1}\right)\right)(\boldsymbol{x}) \\
& =f_{k-1}(\boldsymbol{x})+\left(\mathscr{B}_{N} g\right)(\boldsymbol{x})-\left(\mathscr{C}_{N, \Gamma} f_{k-1}\right)(\boldsymbol{x}) .
\end{aligned}
$$

where

$$
\mathscr{C}_{N, \Gamma} \triangleq \mathscr{B}_{N} \circ \mathscr{D}_{\Gamma}
$$

The convergence analysis can be directly inferred from the time/frequency case presented in [1].

\section{NUMERICAL EXAMPLE}

We apply the proposed method to extrapolate incomplete data on a sphere and examine the algorithm performance. The data is artificially generated by assigning random spherical harmonic coefficients up and excluding order $N=4$.

The observations are given for $0 \leq \theta \leq 140^{\circ}, 0 \leq \phi \leq$ $300^{\circ}$. Fig. 2 shows the original signal, the given observations and the estimated signal after 30 iterations. It can be seen that the algorithm provides very accurate extrapolation results.

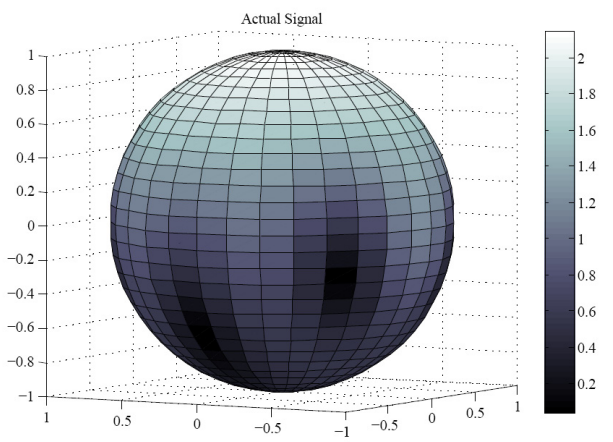

(a) Original signal $f(\theta, \phi)$.
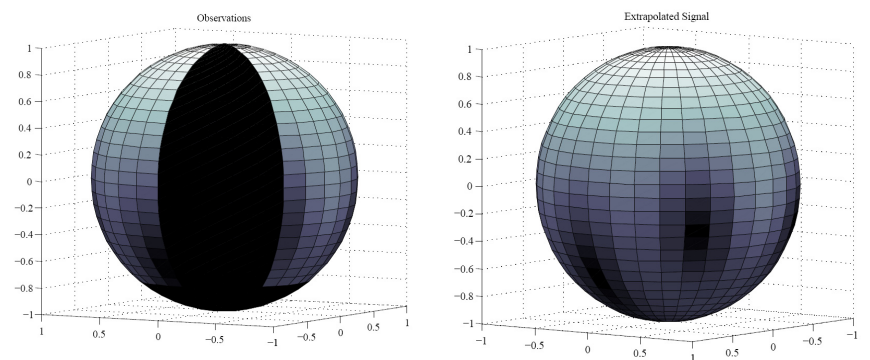

(b) Given Observations $g(\theta, \phi)$ (c) Extrapolated signal $f_{k}(\theta, \phi)$

Fig. 2. An example of data reconstruction on the sphere using the proposed iterative algorithm. The observations in (b) are given for $0 \leq \theta \leq 140^{\circ}$, $0 \leq \phi \leq 300^{\circ}$ with $N=4$. Extrapolation results in (c) are the estimates after 30 iterations. The color scale shows the signal magnitude; and the pure black means no observation made.

\section{Conclusions}

The classical problem of extrapolation of a bandlimited signal from limited time domain data was revisited for signals defined on the sphere. Our investigations explored the role of integral equation operators in characterizing the extrapolation problem which leads to an iterative algorithm analogous to that obtained in the time-frequency case.

\section{REFERENCES}

[1] A. Papoulis, "A new algorithm in spectral analysis and band-limited extrapolation," IEEE Trans. Circuits and Syst., vol. CAS-22, no. 9, pp. 735-742, Sept. 1975.

[2] D. Slepian and H. O. Pollak, "Prolate spheroidal wave functions, fourier analysis and uncertainty - I," Bell Systems Technical Journal, pp. 45-63, 1961.

[3] D. Slepian, "Some comments on fourier analysis, uncertainty and modeling," SIAM Review, vol. 25, no. 3, pp. 379-393, jul 1983.

[4] R. A. Kennedy and T. D. Abhayapala, "Spatial concentration of wave-fields: Towards spatial information content in arbitrary multipath scattering," in Proc. 4th Australian Communications Theory Workshop, Melbourne, Australia, February 2003, pp. 38-45.

[5] F. J. Simons, F. A. Dahlen, and M. A. Wieczorek, "Spatiospectral concentration on a sphere," SIAM Review, vol. 48, no. 3, pp. 504-536, 2006.

[6] W. Zhang, R. A. Kennedy, and T. D. Abhayapala, "Iterative extrapolation algorithm for data reconstructin over sphere," in Proc. IEEE Int. Conf. Acoust., Speech, Signal Processing, ICASSP'2008, Las Vegas, Nevada, March 2008 (to appear).

[7] W. Zhang, R. A. Kennedy, and T. D. Abhayapala, "Signal estimation from incomplete data on the sphere," in Proc. 8th Australian Communications Theory Workshop, AusCTW'2007, Christchurch, NZ, February 2008, pp. 39-44.

[8] D. Colton and R. Kress, Inverse Acoustic and Electromagnetic Scattering Theory, Springer-Verlag, Berlin, Germany, second edition, 1998.

[9] F. Riesz and B. Sz.-Nagy, Functional Analysis, Dover Publications Inc., New York, NY, second edition, 1990.

[10] L. Debnath and P. Mikusiński, Introduction to Hilbert Spaces with Applications, Academic Press, San Diego, CA, 1999, Classic exposition.

[11] M. Nees, "Products of orthogonal projections as Carleman operators," Integral Equations and Operator Theory, vol. 35, no. 1, pp. 85-92, 1999. 